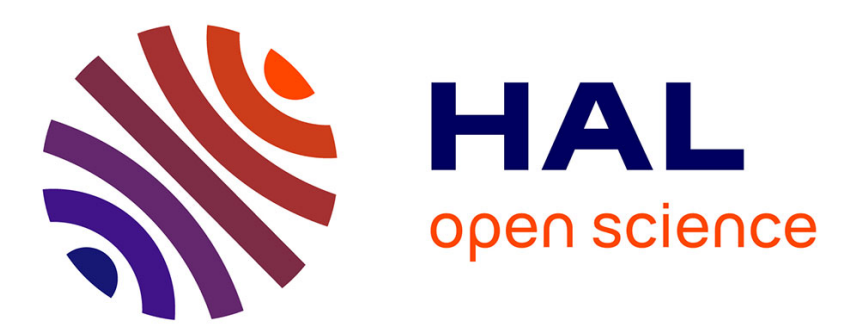

\title{
Application of a two-point closure to the dispersion of particles in isotropic and sheared turbulence
}

Wouter J.T. Bos, Jean-Pierre Bertoglio

\section{To cite this version:}

Wouter J.T. Bos, Jean-Pierre Bertoglio. Application of a two-point closure to the dispersion of particles in isotropic and sheared turbulence. iTi Conference on Turbulence 2005, Sep 2005, Bad Zwischenahn, Germany. pp.217-220, 10.1007/978-3-540-32603-8_44 . hal-00461261

\section{HAL Id: hal-00461261 https://hal.science/hal-00461261}

Submitted on 4 Mar 2010

HAL is a multi-disciplinary open access archive for the deposit and dissemination of scientific research documents, whether they are published or not. The documents may come from teaching and research institutions in France or abroad, or from public or private research centers.
L'archive ouverte pluridisciplinaire HAL, est destinée au dépôt et à la diffusion de documents scientifiques de niveau recherche, publiés ou non, émanant des établissements d'enseignement et de recherche français ou étrangers, des laboratoires publics ou privés. 


\title{
Application of a two-point closure to the dispersion of particles in isotropic and sheared turbulence
}

\author{
Wouter Bos and Jean-Pierre Bertoglio \\ Laboratoire de Mécanique des Fluides et d'Acoustique \\ Ecole Centrale de Lyon, Ecully, France
}

\section{Introduction}

Batchelor [1] was the first to formally describe the link between particle dispersion and the mixing of a passive scalar. Since then, several authors have used this link to address the problem of dispersion. For example, Larcheveque and Lesieur [2] used the Eddy Damped Quasi-Normal Markovian closure (EDQNM) for the scalar spectrum to study the relative dispersion of particle pairs. A recent review of dispersion and mixing can be found in Falkovich et al. [3]. In the present work it is shown that a recent extension of EDQNM to the case of homogeneous turbulence with a uniform mean scalar gradient can be applied to single-particle dispersion in both isotropic turbulence and homogenous shear flow.

\section{Single particle dispersion in isotropic turbulence}

We first consider an isotropic turbulent velocity field on which we impose a constant uniform mean temperature gradient $\Gamma$ in the $z$-direction. The scalar is assumed non-diffusive so that the equation for the scalar fluctuation reads:

$$
\frac{d \theta}{d t}=-\Gamma w^{\prime},
$$

with $w^{\prime}$ the $z$-component of the fluctuating velocity field. It can be easily seen by integrating the scalar equation over a particle trajectory that in this case the scalar fluctuation $\theta$ is directly related to the vertical separation distance $z^{\prime}$ of the particle from its initial position:

$$
\theta=-\Gamma z^{\prime}
$$

The mean squared displacement then obeys the equation: 


$$
\frac{\partial \overline{z^{\prime 2}}}{\partial t}=2 \overline{w z^{\prime}}=-2 \Gamma^{-1} \overline{w \theta}
$$

in which the only unknown quantity is the scalar-velocity cross-correlation $\overline{w \theta}$. Using the EDQNM closure for the scalar-velocity cross-correlation spectrum $[4,5], \overline{w \theta}$ can be evaluated. The resulting predictions for the mean squared displacement are found in good agreement with the classical dispersion theory of Taylor [6]. The results are shown in figure 1.

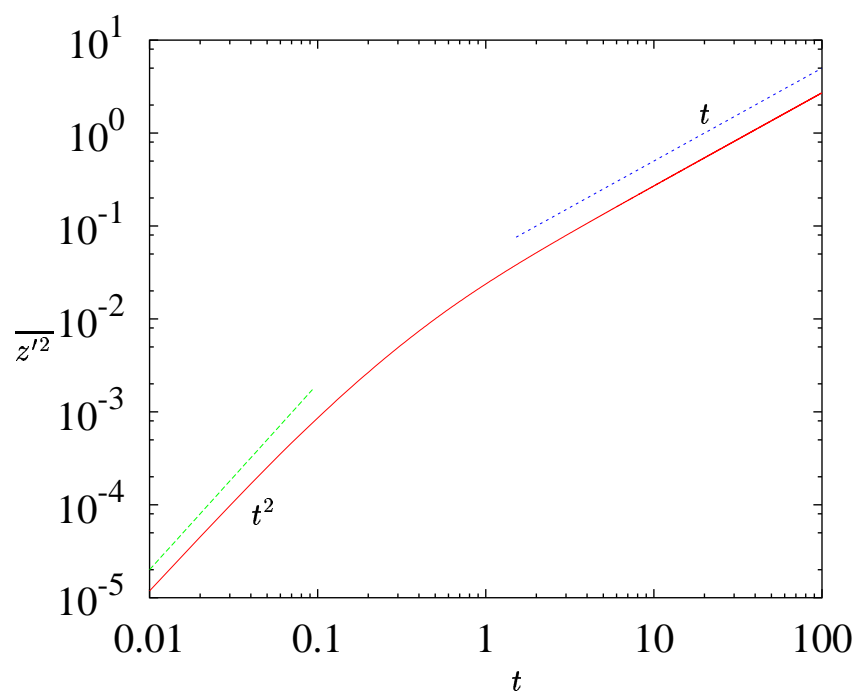

Fig. 1. Ballistic and Brownian regimes of single particle dispersion

\section{Dispersion in homogeneous shear flow}

Taylor predicted a $t^{3}$ behaviour for the horizontal dispersion in stationary homogeneous shear flow. While DNS is very limited to make large time or large Reynolds number predictions, a recent extension of the EDQNM theory to scalar mixing in homogeneous shear flow [7] allows to study the dispersion in horizontal and vertical direction for long times and virtually without limit in Reynolds number.

We consider homogeneous shear flow with a mean velocity gradient $S=$ $\partial U / \partial z$. The vertical displacement obeys the same analogy with a non-diffusive scalar as in isotropic turbulence. The horizontal displacement is however explicitly affected by the mean velocity:

$$
\frac{d x^{\prime}}{d t}=u^{\prime}+z^{\prime} S
$$


with $u^{\prime}$ the $x$-component of the fluctuating velocity field. An additional term has to be added to the scalar equation to obtain a direct analogy. The equations for the scalars $\theta$ and $\chi$ corresponding to the vertical and horizontal displacements are now respectively:

$$
\begin{gathered}
\frac{d \theta}{d t}=-\Gamma_{\theta} w^{\prime} \\
\frac{d \chi}{d t}=-\Gamma_{\chi} u^{\prime}-\theta S
\end{gathered}
$$

In these equations $\Gamma_{\theta}$ is a mean scalar gradient in the $z$-direction and $\Gamma_{\chi}$ is a mean scalar gradient in the $x$-direction. The equations for the mean squared displacement distance read:

$$
\begin{array}{r}
\frac{d \overline{z^{\prime 2}}}{d t}=2 \overline{w^{\prime} z^{\prime}} \\
\frac{d \overline{x^{\prime} z^{\prime}}}{d t}=\overline{u^{\prime} z^{\prime}}+\overline{w^{\prime} x^{\prime}}+\overline{z^{\prime 2}} S \\
\frac{d \overline{x^{\prime 2}}}{d t}=2 \overline{u^{\prime} x^{\prime}}+2 \overline{z^{\prime} x^{\prime}} S
\end{array}
$$

The cross-correlations $\overline{u^{\prime} z^{\prime}}$ and $\overline{w^{\prime} z^{\prime}}$ are again treated as scalar fluxes, in the case of a vertical mean scalar gradient with value -1 . The cross-correlations $\overline{u^{\prime} x^{\prime}}$ and $\overline{w^{\prime} x^{\prime}}$ are treated as scalar fluxes in the presence of a horizontal mean scalar gradient, taking into account the coupling term $(-\theta S)$ in equation (6). The influence of this coupling term is neglected in the equation for the triple correlations. The details of the closure used to obtain quantitative results, based on the EDQNM theory, can be found in Bos [7].

The result is shown in figure 2 . The ballistic regime is present again and after a transitional part a $t^{3}$ behaviour is observed. At the longest times the exponential growth of the kinetic energy in homogenous shear flow causes the dispersion to deviate from this scaling and to grow faster than $t^{3}$.

\section{Conclusion}

The analogy of the equation for the scalar fluctuation in a turbulent flow with a mean passive scalar gradient and the deplacement of a fluid particle allows to use closures developed for passive scalar mixing to obtain results for fluid particle dispersion. The EDQNM closure, developed for the scalar flux, is used without modification in this work to predict single particle dispersion in isotropic turbulence and homogeneous shear flow. The results are in agreement with classical scaling laws. 


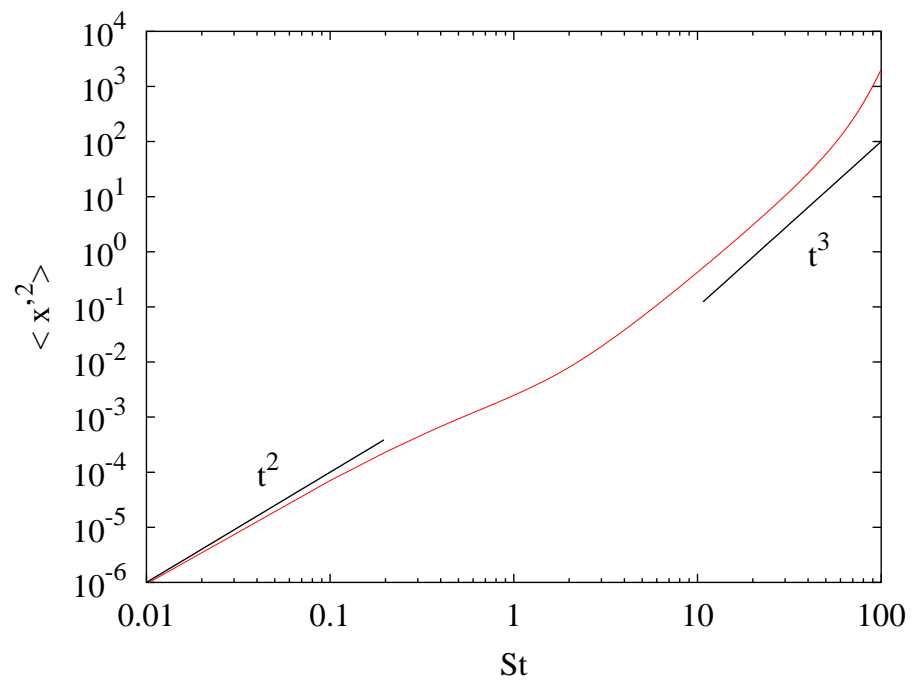

Fig. 2. Horizontal dispersion of a single particle in homogeneous shear flow

\section{References}

1. G.K. Batchelor. Diffusion in a field of homogeneous turbulence. i. eulerian analysis. Aust. J. Sci. Res. Ser. A, 2:437-450, 1949.

2. M. Larchevêque and M. Lesieur. The application of eddy-damped markovian closures to the problem of dispersion of particle pairs. J. Mécanique, 20:113134, 1981.

3. G. Falkovich, K. Gawedzki, M. Vergassola. Particles and fields in fluid turbulence. Rev. Mod. Phys., 73(4):913-975, 2001.

4. S. Herr, L.P. Wang, and L.R. Collins. EDQNM model of a passive scalar with a uniform mean gradient. Phys. Fluids, 8:1588-1608, 1996.

5. W.J.T. Bos, H. Touil, and J.-P. Bertoglio. Reynolds number dependency of the scalar flux spectrum in isotropic turbulence with a uniform scalar gradient. Phys. Fluids, 17(12), 2005.

6. G.I Taylor. Diffusion by continuous movements. Proc. London Math. Soc., 2(20):196-212, 1921.

7. W.J.T. Bos. Passive Scalar mixing in turbulent flow. PhD thesis, Ecole Centrale de Lyon, 2005. 